\title{
Evaluation of a protective effect of in ovo delivered Campylobacter jejuni OMVs
}

\author{
Renata Godlewska $^{1}$ (D) Maciej Kuczkowski ${ }^{2}$ - Agnieszka Wyszyńska ${ }^{1}$ • Joanna Klim² ${ }^{2}$ \\ Katarzyna Derlatka $^{1}$ • Anna Woźniak-Biel ${ }^{2}$ • Elżbieta K. Jagusztyn-Krynicka ${ }^{1}$
}

Received: 31 March 2016 / Revised: 13 June 2016 / Accepted: 19 June 2016 / Published online: 6 July 2016

(C) The Author(s) 2016. This article is published with open access at Springerlink.com

\begin{abstract}
Campylobacter jejuni is the most prevalent cause of a food-borne gastroenteritis in the developed world, with poultry being the main source of infection. Campylobacter jejuni, like other Gram-negative bacteria, constitutively releases outer membrane vesicles (OMVs). OMVs are highly immunogenic, can be taken up by mammalian cells, and are easily modifiable by recombinant engineering. We have tested their usefulness for an oral (in ovo) vaccination of chickens. Four groups of 18-day-old chicken embryos (164 animals) underwent injection of wt $C$. jejuni OMVs or modified OMVs or PBS into the amniotic fluid. The OMVs modifications relied on overexpression of either a complete wt cjaA gene or the C20A mutant that relocates to the periplasm. Fourteen days post-hatch chicks were orally challenged with live C. jejuni strain. Cecum colonization parameters were analyzed by two-way ANOVA with Tukey post-hoc test. The wtOMVs and OMVs with wtCjaA overexpression were found to confer significant protection of chicken against $C$. jejuni $(p=0.03$ and $p=0.013$, respectively) in comparison to PBS controls and are promising candidates for further in ovo vaccine development.
\end{abstract}

Electronic supplementary material The online version of this article (doi:10.1007/s00253-016-7699-x) contains supplementary material, which is available to authorized users.

Renata Godlewska

renatag@biol.uw.edu.pl

1 Department of Bacterial Genetics, Institute of Microbiology, Faculty of Biology, University of Warsaw, Miecznikowa 1,

02-096 Warsaw, Poland

2 Department of Epizootiology and Clinic for Birds and Exotic Animals, Faculty of Veterinary Medicine, Wrocław University of Environmental and Life Sciences, Pl. Grunwaldzki 45, 50-366 Wrocław, Poland
Keywords Campylobacter jejuni - Outer membrane vesicles $(\mathrm{OMV}) \cdot$ in ovo immunization

\section{Introduction}

Campylobacter strains are recognized as a major causal agent of bacterial diarrhea both in developing and developed regions of the world. Campylobacter infection rates have been increasing steadily over the past decade. In EU countries, the number of confirmed human campylobacteriosis cases was 236,851 in 2014 , an increase of 22,067 cases (9.6\%) compared to 2013 (EFSA and ECDC 2015). Mortality associated with Campylobacter infections is relatively low, and no specific treatment is required for a vast majority of patients. However, Campylobacter infections constitute a serious problem due to the high number of cases, severity of possible neurological complications, as well as high social and economic costs of the disease (Kaakoush et al. 2015; Kirkpatrick and Tribble 2011).

The consumption of infected poultry meat is a major source of infection. European Food Safety Authority (EFSA) reported that in 2014, $38.4 \%$ of the 6703 samples of fresh broiler meat were found to be Campylobacter positive (EFSA and ECDC 2015). Efforts to comply with EU hygiene and biosecurity regulations appear insufficient to control or eliminate Campylobacter from the poultry food chain (Havelaar et al. 2007; Mangen et al. 2007). Elimination of Campylobacter jejuni from chickens would significantly reduce the incidence of campylobacteriosis in humans and seems to be an alternative and more realistic approach for controlling Campylobacter contamination. However, antiCampylobacter chicken vaccines are not commercially available yet.

Many Gram-negative bacteria, especially pathogenic ones (such as Neisseria meningitidis, Haemophilus influenzae, 
Helicobacter pylori, Borrelia burgdorferi, Pseudomonas aeruginosa, Shewanella spp., Shigella spp., Salmonella spp.), produce outer membrane vesicles (OMVs). Secretion of these vesicles to a medium is a naturally occurring phenomenon. Vesicles contain not only outer membrane-associated proteins but also periplasmic and even cytoplasm-located molecules (virulence factors, DNA chains, enzymes) (Kuehn and Kesty 2005).

Considering their immunogenic and self-adjuvant properties, the ability to be taken up by mammalian cells, and adjustability of their content by recombinant engineering, OMVs are attractive candidates for vaccine delivery vectors. Composition of the OMVs (LPS, glycerophospholipids, OM and periplasmic proteins) makes them capable of mediating both pro- and antiinflammatory activities leading to a clearance of infection or a widespread inflammation (Mashburn-Warren et al. 2008; Mashburn and Whiteley 2005). That is why OMVs are useful as vaccines and adjuvants stimulating protective mucosal and humoral immune responses (Collins 2011), already constituting a base of several licensed vaccines and gaining an increasing popularity (van der Pol et al. 2015). For example, conventional wild-type outer membrane vesicle (wtOMV) vaccines are the only formulations that have shown efficacy against serogroup B meningococcal disease (Acevedo et al. 2014; Asensio et al. 2011; Holst et al. 2009; Roberts et al. 2008). Consequently, a novel vaccine (4CMenB), against serogroup B meningococcal disease, composed of protein antigens identified by reverse vaccinology (fHBP fused to GNA2091, GNA2132 fused to GNA1030, and NadA), combined with OMVs is now approved in Europe, Canada, Australia, and some Latin American countries (Carter 2013; Giuliani et al. 2006; Martin and Snape 2013; Serruto et al. 2010).

Campylobacter jejuni OMVs have not yet attracted a comparable interest. It has only been shown that $C$. jejuni membrane vesicles contain a toxin-dubbed cytolethal distending toxin (CDT) (Elmi et al. 2012; Lindmark et al. 2009), and proteomic analyses of $C$. jejuni OMVs identified 151 proteins, including periplasmic, outer membrane associated, innermembrane, and even cytoplasmic ones. Among them, all three subunits of CDT (CdtA, CdtB, and CdtC) and sixteenglycosylated proteins were present (Elmi et al. 2012; Jang et al. 2014).

One of the latter is $\mathrm{CjaA}$, an extracytoplasmic, glycosylated and highly immunogenic protein cloned and characterized in our laboratory (Pawelec et al. 1997; Wyszynska et al. 2008). CjaA is conserved between different Campylobacter serotypes. Crystallographic analyses of the Escherichia coli-produced rCjaA determined that $\mathrm{CjaA}$ binds to the cysteine ligand and is a constituent of the $\mathrm{ABC}$ cysteine transport system (Muller et al. 2005). Expression of $\mathrm{CjaA}$ in bacteria cultured on solid media increases under iron deficiency conditions and is higher in clinical Campylobacter isolates than in laboratory strains (Cordwell et al. 2008; Holmes et al. 2005; Shoaf-Sweeney et al. 2008). These facts suggest that $\mathrm{CjaA}$ participates in a colonization process in vivo.

$\mathrm{CjaA}$ is recognized by chicken maternal antibodies (Cordwell et al. 2008; Shoaf-Sweeney et al. 2008), and several groups attempted to utilize it as an antigen for immunization of chickens. We were first to show that vaccination with live $S$. enterica sv. Typhimurium $\chi 3987$-expressing $\mathrm{CjaA}$ reduces the colonization with heterologous $C$. jejuni strain by as much as six logs (Wyszynska et al. 2004). Oral immunization of SPF birds with $S$. enterica sv. Typhimurium aroA mutant producing $\mathrm{CjaA}$ antigen fused to the $\mathrm{C}$-terminal part of the fragment $\mathrm{C}$ of tetanus toxin resulted in about $1.4 \log 10 \mathrm{CFU} / \mathrm{g}$ reduction in the cecal load of $C$. jejuni (Buckley et al. 2010). Similarly, Salmonella-mediated delivery of the linear epitope of $\mathrm{CjaA}$ fused to the LamB protein and coexpressed with the immuneenhancing CD154 ligand caused approximately 2-log reduction in ileal load of $C$. jejuni (Layton et al. 2011). Finally, oral inoculation of SPF chicken with Eimeria tenella oocysts, modified to express CjaA on the surface, induced 91 and $86 \%$ immune protection against $C$. jejuni challenge compared with unvaccinated and wild-type E. tenella-vaccinated controls (Clark et al. 2012). All these experiments demonstrated a promising, albeit not sufficient protection; importantly, the effect was dependent also on chicken breeds used.

In this study, we employed yet another delivery mode and evaluated the protective effect of a nonadjuvanted, multivalent OMV vaccine administered in ovo against infection with C. jejuni, employing natural OMVs as well as OMVs enriched with $\mathrm{CjaA}$ due to the introduction of an extra copy of the wt cjaA gene or C20A mutant of the cjaA known to shift the protein localization into periplasm. Our work demonstrated that the immunization of chickens with OMVs provides a mean to reduce a cecal colonization with wt $C$. jejuni.

\section{Materials and methods}

\section{Bacterial strains, media, and culture conditions}

Bacterial strains and plasmids used in this study are listed in Table 1.

Campylobacter jejuni strains were grown on Blood Agar no. 2 (BA, Oxoid) plates supplemented with $5 \%$ horse blood, Campylobacter selective supplement (Blaser-Wang) (Oxoid, Basingstoke, UK), and tetracycline $(10 \mu \mathrm{g} / \mathrm{ml})$ at $37{ }^{\circ} \mathrm{C}$ or $42{ }^{\circ} \mathrm{C}$ for $16-24 \mathrm{~h}$ under microaerobic conditions $\left(6 \% \mathrm{O}_{2}\right.$, $10 \% \mathrm{CO}_{2}, 85 \% \mathrm{~N}_{2}$ ). If necessary, plates were also supplemented with chloramphenicol $(20 \mu \mathrm{g} / \mathrm{ml})$ and/or kanamycine (25 $\mu \mathrm{g} / \mathrm{ml})$. Campylobacter strains used for isolation OMVs were grown in Mueller-Hinton broth under microaerobic conditions at $37^{\circ} \mathrm{C}$. 
Table 1 Strains and plasmids used in this study

\begin{tabular}{|c|c|c|}
\hline Strain or plasmid & Genotype/resistance/description & Reference \\
\hline \multicolumn{3}{|l|}{ Campylobacter jejuni } \\
\hline $\begin{array}{l}81176 \\
(\text { ATCC BAA-2151) }\end{array}$ & Wild type, human isolate & Korlath et al. (1985) \\
\hline $81176 c^{\prime} a A^{-}$ & pVir, cjaA::aphA3, $\mathrm{Tc}^{\mathrm{R}}$ & This study \\
\hline $12($ PCM 2852) & Wild type; isolated from a chicken, good colonizer & Wyszynska et al. (2004) \\
\hline $12 / 2$ & $\begin{array}{l}\text { Wild type; isolated from a chicken with introduced } \\
\text { pUOA18 plasmid; good colonizer, } \mathrm{Cm}^{\mathrm{R}}\end{array}$ & (Wyszynska et al. 2004) \\
\hline \multicolumn{3}{|l|}{ Escherichia coli } \\
\hline E. coli $\mathrm{S} 17.1$ & $\begin{array}{l}\mathrm{F}^{-} \text {recA thi pro hsd } \mathrm{R}^{-} \mathrm{M}^{+} \mathrm{RP} 4: 2-\mathrm{Tc}: \mathrm{Mu}: \mathrm{Km} \mathrm{Tn} 7 \mathrm{Tp}^{\mathrm{R}} \\
\mathrm{Sm}^{\mathrm{R}} \lambda \text { pir } \operatorname{Tra}^{+}\end{array}$ & Simon et al. (1983) \\
\hline \multicolumn{3}{|l|}{ Plasmids } \\
\hline pUWM639 & cjaA gene (from 81176) cloned into pRY111, $\mathrm{Cm}^{\mathrm{R}}$ & This study \\
\hline pUWM1405 & $\begin{array}{l}\text { cjaA gene (from } 81176 \text { ) with } \mathrm{C} 20 \mathrm{~A} \text { point mutation } \\
\text { cloned into } \mathrm{pRY} 111, \mathrm{Cm}^{\mathrm{R}}\end{array}$ & This study \\
\hline
\end{tabular}

Campylobacter jejuni 12/2 strain, isolated from an intestinal tract of a chicken, labeled with the pUOA18 plasmid containing cat gene, was utilized in challenge experiments.

\section{Site-directed mutagenesis}

Point mutation was generated using the QuikChange sitedirected mutagenesis kit according to procedures recommended by the supplier (Stratagene). A derivative of pBluescript II SK, containing the $c j a A$ gene with its own promoter from $C$. jejuni $81176 \mathrm{pVir}$, was used as a template for PCR-mediated mutagenesis. Point mutation $\mathrm{C} 20 \mathrm{~A}$ was introduced with primers CAGTAGTATTGGCTGCTGCTGGAGGAAATTCTGACTCTAAAAC and GTTTTAGA $\overline{\bar{G}}$ TCAGAATTTCCTCCAGCAG CAGCCAATACTACTG (mismatches are double underlined).

The plasmid containing the $c j a A$ gene with $\mathrm{C} 20 \mathrm{~A}$ was transformed into $E$. coli TG1, and a presence of the desired mutation was verified by DNA sequencing. After digestion of the resulting recombinant plasmid with NotI and SalI, the fragment containing the $c j a A$ gene was cloned into the shuttle vector $\mathrm{pRY} 111$ digested with the same enzymes. The resulting plasmid was named pUWM1405 (cjaA with C20A mutation). Shuttle plasmid containing a wild copy of the $C$. jejuni cjaA gene was named pUWM639. All derivatives of pRY111 were introduced into $C$. jejuni $c j a A^{-}$by bi-parental conjugation (Davis et al. 2008).

We had to recreate the $C$. jejuni $81176 c j a A^{-}$mutant. The one used in our previous studies had lost pTet plasmid, which could be important in conjugative transfer of plasmids. New mutant was constructed using standard natural transformation assay (Vegge et al. 2012). Exponentially, growing cells of $C$. jejuni $81176 \mathrm{Tc}^{\mathrm{R}}$ were transformed with genomic DNA of $C$. jejuni $81176: \mathrm{CjaA}^{-}, \mathrm{Tc}^{\mathrm{S}}$ strain. The disruption of $c j a A$ gene was confirmed by PCR and sequencing.

\section{OMV purification and quantitation}

OMVs were isolated from C. jejuni strain 81176 using methods described by Elmi et al. (2012) and Chutkan et al. (2013).

Campylobacter jejuni 81176 strain was grown in the Mueller-Hinton broth under microaerobic conditions at $37^{\circ} \mathrm{C}$. An overnight culture was diluted 1:100 into $330 \mathrm{ml}$ of fresh growth media and grown $16-18 \mathrm{~h}$ to a mid log phase. Briefly, the cells were pelleted twice, using centrifugation at $6000 \times g$ for $2 \times 20 \mathrm{~min}$ at $4{ }^{\circ} \mathrm{C}$. Supernatants were filtered through a $0.22-\mu \mathrm{m}$ filter device to remove remaining cells. The filtrate was ultracentrifuged in Beckman L7-55 Ultracentrifuge at $150,000 \times \mathrm{g}$ for $3 \mathrm{~h}$ at $4{ }^{\circ} \mathrm{C}$, using a $50.2 \mathrm{Ti}$ rotor. OMV preparations were plated on BA plates and incubated under microaerobic conditions to confirm the absence of viable bacteria.

The pellets were resuspended with Dulbecco's phosphatebuffered saline (DPBS) or PBS and stored at $-20{ }^{\circ} \mathrm{C}$ until needed. Before injecting into eggs, the OMV samples were sterilized by filtration using Ultrafree-MC-GV Centrifugal filters (Merck Millipore). A protein content of OMVs was measured by BCA assay.

\section{Enrichment of $C$. jejuni OMVs with the CjaA protein}

Plasmid pUWM639, carrying an additional copy of $c j a A$ gene on the shuttle vector (pRY 111 plasmid), was introduced into 81176 C. jejuni $\mathrm{cjaA}^{-}$strain by standard bi-parental conjugation procedure (with E. coli S17.1 strain) (Davis et al. 2008). Similarly, pUWM1405, with C20A point mutation in cjaA gene, was introduced into 81176 C. jejuni cjaA $^{-}$to increase the $\mathrm{CjaA}$ content in periplasm. The OMVs produced by C. jejuni 81176/ pUWM639 and $C$. jejuni 81176/pUWM1405 strains were purified as described above. The quantification of $\mathrm{CjaA}$ protein in OMV from abovementioned strains was carried out using enzyme-linked immunosorbent assay (ELISA). Serial dilutions 
of total, heat-treated $\left(100{ }^{\circ} \mathrm{C}\right.$ for $\left.20 \mathrm{~min}\right)$ OMVs were used to coat 96-well plates. The recombinant $\mathrm{CjaA}$ antigen was used as the plate-coating antigen for construction of a standard curve. Coated wells were incubated with specific rabbit anti-rCjaA antibodies and with alkaline phosphatase-conjugated second goat anti-rabbit IgG antibodies (Sigma-Aldrich). The reaction was run with $p$-nitrophenyl phosphate $(1 \mathrm{mg} / \mathrm{ml})$ as a substrate and was stopped after 30-min incubation (room temperature) with 3.0-M sodium hydroxide. Optical density was determined at $405 \mathrm{~nm}$ using the ELISA reader (Tekan). Each sample was analyzed in triplicate. The results were expressed relative to the standard protein per milligram of total OMV protein.

\section{Animal supply and housing}

Animal experiments were performed using the Rosal breed of chickens. This breed was created by crossing Sussex hen with Rhode Island Red rooster. The birds were housed in separate cages for each group and given water and food ad libitum. All animal experiments were carried out with approval nr 397/ 2012 of the LKE (First Local Ethical Committee on Animal Testing in Warsaw).

\section{Immunization and challenge regimen}

One hundred sixty-four 18-day-old chick embryos of laying hens (Rosal breed) were orally immunized by injection of 0.1-ml different OMVs (wtOMVs, 639-OMVs, 1405OMVs) into the amniotic fluid. Each injection dose contained $200 \mu \mathrm{g}$ of protein. Groups of birds inoculated with PBS buffer served as controls. Fourteen-day post-hatch half of all chicks groups were orally challenged with $10^{5}$-CFU of the live C. jejuni $12 /$ pUOA18 strain. Cloacal swabs were taken every 2 days after hatch to evaluate Campylobacter colonization of birds. After the challenge, the $C$. jejuni present in chicken cecal contents were enumerated by plating. On 21 and 28 days after hatch, chicks were sacrificed, and their cecal contents were aseptically removed. Samples were weighed and nine times their weight of PBS was added. Samples were homogenized, and serially diluted. $0.1 \mathrm{ml}$ of each dilution was plated on Campylobacter BA plates, supplemented with chloramphenicol. The scheme of the protection experiment is shown in Table 2.
Samples of intestinal content and blood were collected on 2, 7, 14, 21 and 28-day post-hatch as shown in Table 2. ELISA test was performed to determine the level of $C$. jejuni-specific immunoglobulin Y (IgY) antibodies and immunoglobulin A $(\operatorname{Ig} \mathrm{A})$ in serum and intestinal secretion samples.

\section{ELISA for serum IgG antibodies and intestinal IgA antibodies}

The level of antibodies against $C$.jejuni protein in intestinal secretions and sera of chicken was quantified by ELISA.

Ninety-six-well plates (Nunc, Thermo Scientific) were coated overnight at $4{ }^{\circ} \mathrm{C}$ with whole-cell $C$. jejuni proteins (20 $\mu \mathrm{g} /$ well), washed three times with PBS containing $0.02 \%$ Tween 20 (Sigma-Aldrich), blocked for $1 \mathrm{~h}$ at $37{ }^{\circ} \mathrm{C}$ with PBS containing $2 \%$ bovine serum albumin (SigmaAldrich), washed as described previously, and incubated for $1.5 \mathrm{~h}$ at room temperature with either diluted sera $(1: 300)$ or intestinal secretion samples (1:10). The plates were developed with 3,3',5,5'-tetramethylbenzidine (Sigma-Aldrich), using goat anti-chicken IgA horseradish peroxidase conjugate (Thermo Scientific, Rockford, IL) or rabbit anti-chicken IgY (whole molecule)-peroxidase (Sigma-Aldrich) (dilution 1:2000), respectively. The plates were incubated with the substrate for $25 \mathrm{~min}$ at room temperature and then the colorimetric reaction was stopped by adding 2- $\mathrm{M} \mathrm{H}_{2} \mathrm{SO}_{4}$ (SigmaAldrich). Absorbance was measured at $490 \mathrm{~nm}$ using an ELISA reader (Tekan). Each sample was analyzed in duplicate.

\section{Statistical analyses}

All statistical analyses of the colonization results were performed using GraphPad Prism 6 (GraphPad Software, San Diego, CA) or STATISTICA 10PL software (StatSoft, USA). The significance of differences between the obtained values was appraised using one-way analysis of variance (ANOVA), followed by the post-hoc Tukey's tests. Statistical analyses of ELISA test were assessed using multifactorial (two-way) ANOVA nested for duplicates and Scheffe's post-hoc test or the Kruskal-Wallis test followed by Dunn's multiple comparison post-hoc test. Any $p$ values $<0.05$ were considered significant.
Table 2 Scheme of immune response and protection experiments

\begin{tabular}{lllllll}
\hline Week of life & -1 & 0 & 1 & 2 & 3 & 4 \\
\hline Immunization with OMVs & + & & & & & \\
Collection of gut secretion and blood samples for immune response analysis & & + & + & + & + & + \\
Challenge with Campylobacter & & & & + & + \\
Cecum isolation for Campylobacter enumeration & & & & + & + \\
\hline
\end{tabular}




\section{Results}

\section{Enrichment of $C$. jejuni OMVs with the CjaA protein}

We decided to utilize OMVs for in ovo immunization of chickens, additionally attempting to enrich OMVs with extra $\mathrm{CjaA}$ in order to increase the load of the antigen in the vaccine. Two strategies were employed toward this goal. The first one relied on expressing an additional copy of the complete $c j a A$ gene introduced into the multicopy plasmid shuttle vector pRY111 (Yao et al. 1993). The constructed plasmid was named pUWM639. Since cjaA expression from pUWM639 was driven by its own strong promoter, it seemed plausible that this manipulation would increase the content of $\mathrm{CjaA}$ protein inside the cells and within OMVs. The second strategy took advantage of the fact that $\mathrm{CjaA}$ in Campylobacter cells localizes mainly in the inner membrane (IM). We had previously created a Campylobacter coli 72Dz/92 strain with the $\mathrm{C} 20 \mathrm{~A}$ (cysteine into alanine) point mutation in the $\mathrm{CjaA}$ signal sequence. Lack of this cysteine shifted the location of the protein from inner membrane to periplasm (Wyszynska et al. 2008). We expected that the periplasmic location of CjaA will further increase the quantity of this protein in OMVs. Toward this end, the pUWM1405 plasmid was prepared that contained the C20A cjaA mutant introduced into pRY111.

Both pUWM639 and pUWM1405 plasmids were introduced into $C$. jejuni $81176 c j a A^{-}$strain via conjugation. To ensure an easy detection of the introduced gene expression, the host strain had been previously deprived of endogenous CjaA protein by means of a gene knockout. Western blot experiments with specific rabbit anti-rCjaA antibodies showed a high level of protein production (Fig. S1, supplementary materials).

ELISA assay of the CjaA protein in OMVs isolated from wt strain and 81176 C. jejuni strains harboring pUWM639, and pUWM1405 plasmids demonstrated that OMVs produced by modified strains harbored much higher a content of CjaA antigen than wt OMVs. The results are shown in Fig. 1.

\section{Immunization procedure. Antibody immune response in vaccinated chickens}

A multiantigenic nature of an OMV vaccine may enhance specific humoral immune response to provide protection against $C$. jejuni strains. Therefore, the antibody response of chicks to OMVs delivered in ovo was analyzed. Eighteenday-old embryos were divided into four groups (one control and three treatment groups) and orally immunized by OMVs injection into an amniotic fluid. The control group received sterile PBS. The treatment groups were immunized with wtOMV, 639-OMV, or 1405-OMV. Several experimental studies have shown that in commercial flocks, chicks are

\section{CjaA content in OMV}

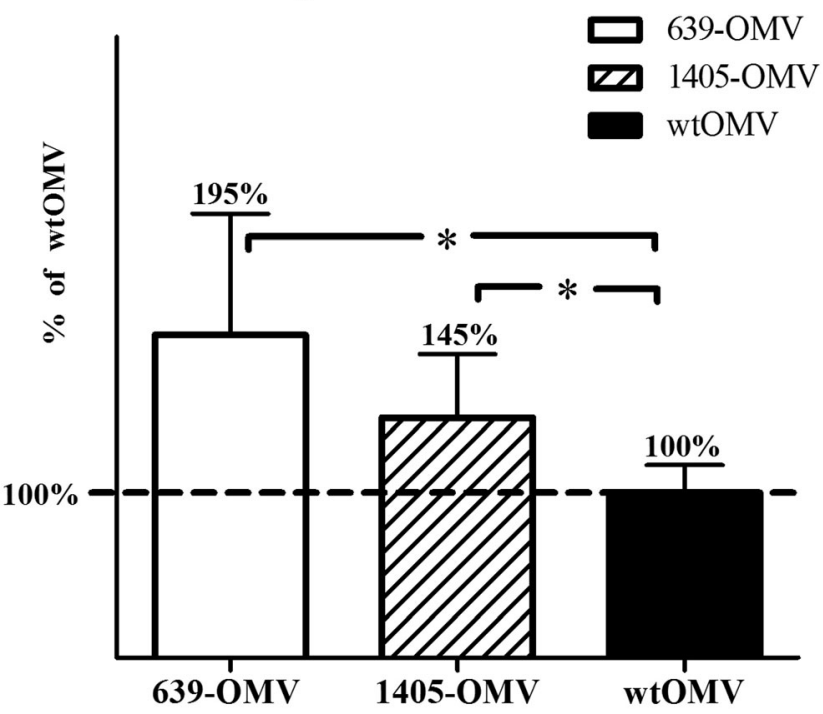

Fig. 1 Comparison of protein CjaA content in different types of OMVs. $\mathrm{CjaA}$ in wtOMV was marked as $100 \%$. The quantification of $\mathrm{CjaA}$ protein in OMV-639, OMV-1405, and wtOMV was carried out using ELISA assay. Serial dilutions of total OMVs were used to coat 96-well plates. The recombinant CjaA antigen was used as the plate-coating antigen for construction of a standard curve. Coated wells were incubated with specific rabbit anti-rCjaA antibodies and with alkaline phosphataseconjugated second goat anti-rabbit IgG antibodies. Each sample was analyzed in triplicate. The results were expressed relative to the standard protein per milligram of total OMV protein. A statistical analysis was carried out using multifactorial (one-way) ANOVA followed by Tukey's multiple comparisons test. Asterisks indicate significant differences $(p<0.05)$ between analyzed groups and the control group

colonized by $C$. jejuni around second or third week of life (Conlan et al. 2007). Therefore, 2 weeks after hatching, half of the chicks from each group were challenged with $10^{5}$ bacterial cells of a chicken-isolated C. jejuni strain. Serum IgY and mucosal IgA antibody responses against Campylobacter proteins were measured by ELISA assay using the whole-cell lysate as a coating antigen. Analysis of serum samples obtained from 1-, 7- to 14-day-old chicks showed relatively high levels of specific IgY in all groups, which dramatically decreased by week 2 . Starting at 1 week after challenge, antiCampylobacter IgY antibody level consistently increased in all experimental groups (data not shown). IgY antibody titers did not differ significantly among the four experimental groups at any of the time points (Kruskal-Wallis test).

Analysis of the intestinal samples also showed an induction of specific mucosal anti-Campylobacter IgA antibodies (Fig. 2). We observed an increasing level of IgA antibody 2 weeks after challenge ( 28 days of life), but the differences again were not statistically significant.

Multifactorial (two-way) ANOVA nested for duplicates of ELISA measurement indicated that IgA levels in challenged animals significantly correlated with time and OMVs type 


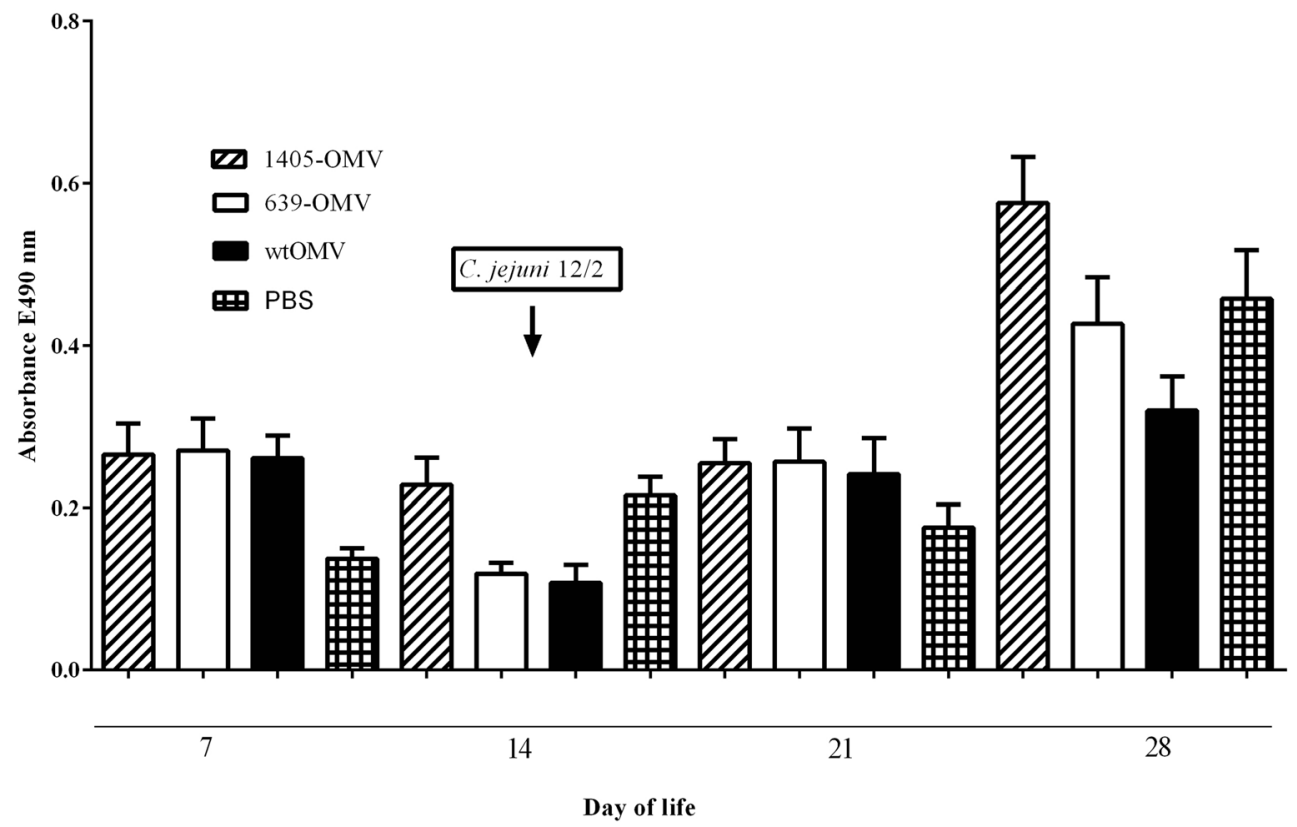

Fig. 2 Mean anti-Campylobacter-specific mucosal IgA levels measured by ELISA at the time intervals on the $\mathrm{x}$-axis. Various types of OMVs were administred in ovo to 18-day-old chickens embryos. Intestinal samples (ceca) were collected, when birds were 1, 2, 3, and 4 weeks old. Campylobacter whole-cell lysates were used as coating antigens.

( $p<0.00001$ and $p<0.0009$, respectively), and there was also a significant interaction of time and OMV type $(p<0.004)$. Overall, there was a very significant increase of $\operatorname{IgA}$ at day 28 $(p<0.000001)$ and a slight decrease at day 14 compared to day 7 ( $p=0.024$; Scheffe's post-hoc tests). Overexpression of a C20A mutant of CjaA was the only manipulation significantly affecting IgA level, leading to a slight increase in comparison to PBS and wt treatment ( $p<0.02$ and $p<0.004$, respectively, Scheffe's post-hoc test) in univariate analysis; for interactions, expression of a C20A mutant led to a very significant increase of $\operatorname{IgA}$ at day 28 comparing to most other combinations, other changes were nonsignificant or meaningless at best.

\section{Protective efficacy of OMVs in ovo immunization of chickens}

Three different types of OMVs, namely wtOMVs, 639OMVs, and 1405-OMVs were tested for protective effect in Rosa1 chickens. In this study, 18-day-old chicken embryos were in ovo vaccinated with a single dose of OMVs vaccine by oral routes and challenged 2 weeks after hatch with the heterologous wild-type strain marked by plasmid pUOA18. As shown in Fig. 3, the in ovo immunization with $10^{5}$-CFU/ $\mathrm{ml}$ of $C$. jejuni strain $(12 / 2)$ provided a moderate protection against Campylobacter infection at the seventh day after challenge in two vaccinated groups (wtOMV and 639-OMV, $p<0.05$ ). Our results indicated that mean colonization levels
Intestinal secretion samples were diluted 1:10. Error bars indicate standard error of the mean. Statistical analyses of ELISA test were assessed using multifactorial (two-way) ANOVA followed Scheffe's post-hoc test. Statistical analyses were performed using STATISTICA 10PL software (StatSoft, USA). Any $p$ values $<0.05$ were considered significant

were of $2 \times 10^{9} \mathrm{CFU}$ in control group and from $\sim 9 \times 10^{7}$ in 639-OMV group to $3 \times 10^{8}$ in wtOMV and 1405 -OMV groups. Two weeks after challenge, the colonization level of immunized bird's ceca was still lower than nonimmunized bird's ceca, but the results for immunized groups were not statistically significant (Fig. 3).

Multifactorial (two-way) ANOVA on log-transformed CFU data demonstrated that both the time from the challenge and the type of OMVs were independently correlating to colonization level ( $p<004$ and $p<0.007$, respectively). The impact of time indicates an increasing level of infection after 2 weeks compared to 1-week post-challenge. The impact of vaccination with OMVs was further analyzed post-hoc using Tukey's test, revealing that OMVs from wt strain or strain overexpressing wtCjaA confer significant protection.

There was no indication of significant interactions between the time from the challenge and the type of OMV used $(p>0.44)$.

\section{Discussion}

Diverse strategies have been applied to produce an effective anti-Campylobacter vaccine for chickens. The greatest progress has been made in the development of subunit vaccines composed of Campylobacter proteins, whether delivered directly or by vectors such as live-attenuated Salmonella cells expressing Campylobacter antigens (Buckley et al. 2010; 


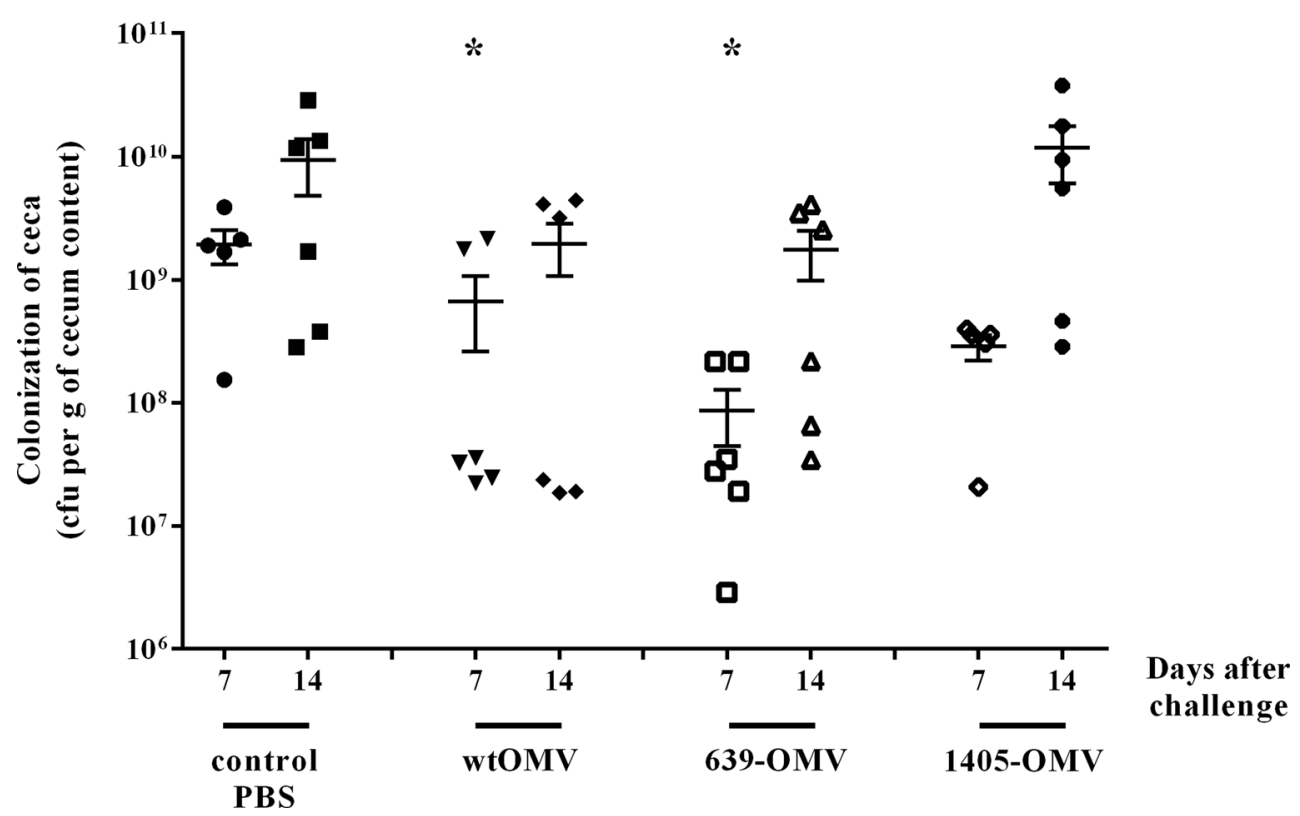

Fig. 3 Colonization of chickens vaccinated with $C$. jejuni OMVs: wtOMV, 639-OMV, and 1405-OMV after $C$. jejuni challenge. Eighteenday-old chicken embryos were given one dose of OMVs and 2 weeks after hatching was challenged with $C$. jejuni 12/2. Control birds were given PBS. Viable $C$. jejuni cells were recovered from the ceca of chickens 7 and 14 days post-infection (d.p.i.). Bacterial recoveries

Layton et al. 2011; Wyszynska et al. 2004). However, the reallife results of their usage are mediocre at best, and new approaches are clearly needed.

The major goal of this study was, therefore, to analyze the potential of OMVs derived from C. jejuni as a new vaccine compound. Here, for the first time, we combined OMVs as a carrier of antigens with in ovo vaccination as a delivery mode to obtain an alternative method for post-hatch immunization of chickens against $C$. jejuni.

In ovo antigen delivery stimulates both innate and adaptive immune responses and can be used for vaccination (especially against viral diseases), for beneficial modification of the bacterial profile in the colon of chickens, for stimulation of an immunological response and embryonic development, and for testing of teratogenic effects (Bande et al. 2015; Negash et al. 2004; Toro et al. 2007).

The avian immune system begins to develop early during embryogenesis, and antibody responses to antigens could be induced in 12-to-14-day-old chicken embryos (Sharma 1999). However, a full immunocompetence normally builds up only after few days of post-hatching (Mast and Goddeeris 1999). Earlier on, the immune defense is provided by maternal antibodies, which had been transferred from a hen to offsprings through egg yolk. Thus, the few days after hatching are crucial: chicks become exposed to environmental threats but are no longer supplemented with new maternal antibodies. And although the gut-associated lymphoid tissue (GALT), which provides an important enteric protection, matures functionally represent colonization levels of five or six birds per time interval. A geometric mean for each group is denoted by a bar. A statistical analysis was carried out using multifactorial (two-way) ANOVA followed by Tukey's multiple comparisons test. Asterisks indicate significant differences $(p<0.05)$ between analyzed groups and the control group

around fourth day after hatching, the secretory IgA response against enteric antigens mounts slowly, completing toward the end of the second week of age (Bar-Shira et al. 2003).

The in ovo vaccination can fill this gap, generating immunity as early as possible, even by the time of hatch.

In order to further improve the in ovo vaccination, we have established and employed a simple way to enrich OMVs with the CjaA antigen based on simple recombinant DNA techniques. We believe that this method is applicable to many other antigens.

Since the protective role of antibodies is regarded as a key factor in the development of poultry vaccines against Campylobacter, we investigated the IgY and IgA levels in chicken from control and immunized groups. Our results are consistent with earlier reports. Sera of chickens contained large quantities of maternal IgY during the first week of life, which decreased gradually during next 2 weeks and resumed to rise after a colonization of the intestinal tract by $C$. jejuni. However, $\operatorname{IgY}$ antibodies play little role in the clearance of $C$. jejuni from cecum, a decrease in the number of $C$. jejuni organisms colonizing the intestinal tract has been observed (Newell and Fearnley 2003; Sahin et al. 2003; ShoafSweeney et al. 2008).

Mucosal IgA is responsible for preventing entry of commensal bacteria into subepithelial areas by blocking their adhesion to epithelial cells or by returning those bacteria that have already penetrated to the basolateral site, without eliciting an inflammatory response (Brisbin et al. 2008). 
They probably play an important role in redirecting inflammatory response toward tolerance. As in the case of IgY, we observed increasing levels of IgA antibodies starting at 1 week after C. jejuni challenge, which proves that Campylobacter challenge triggers birds immune response. In both experiments, the level of mucosal IgA and IgY in serum of noninfected was lower compared to colonized chicken.

Immunization with wtOMVs and OMVs enriched in the CjaA antigen had a significant effect on the cecal load of C. jejuni 7 days after challenge, when compared to vaccination with control preparation. Fourteen days after challenge, the C. jejuni cecal loads were still reduced; however, the differences were not statistically significant anymore. It is puzzling why the periplasmic mutant of CjaA (1405) failed to confer a protection comparable to ones obtained with OMVs from wt and 639 strains. We have verified (Fig. 1) that the expression of this mutant in OMVs was actually higher than wt protein (albeit lower than overexpressed wt protein). This result was obtained using ELISA with polyvalent anti-CjaA rabbit serum. It is possible that the membrane protein, forcefully relocated (released) to periplasm, does not retain (or does not achieve) its normal conformation and/or modifications. Regarding the latter, this mutant remains to appear as a double band on western blotting, which is demonstrated to reflect the glycosylation that it is normally subjected to (Wyszynska et al. 2008). However, this does not preclude that the glycosylation is incomplete/atypical or that the protein is otherwise altered, thus having a distorted antigenicity. As a result, it would be less efficiently detected in ELISA by a polyvalent serum raised against wt protein (leading to false impression of 'decreased' level comparing to overexpressed wt protein), but more importantly, it would elicit an immune response shifted toward 'false' antigens, thus offering decreased protection against wt strains presenting only a typical wt CjaA. This explanation remains speculative as it was not further investigated.

Thus, a single vaccination regime for 18-day-old embryos has induced a protection against $C$. jejuni challenge, but the protection was too short lived. Efforts should be undertaken to enhance OMV vaccine efficacy by combining OMV with appropriate adjuvants or additional protein antigens like in 4CMenB in anti- $N$. meningitidis vaccine (Carter 2013; Martin and Snape 2013). Nevertheless, most of the recently tested vaccines required two doses for a full protection. Thus, the additional dose of a vaccine administrated after hatch may still be needed.

The time and route of administration of such a second dose deserve consideration. Presumably, the booster dose should be given when the defense system is well developed, which is about 7 days after hatch (Mast and Goddeeris 1999).

Regarding the route, in ovo vaccines are typically injected into the amniotic sac. The embryo then swallows and ingests surrounding amnion fluid containing vaccine antigens. Antigens subsequently pass into the intestine, where they can stimulate the immunocompetent cells of GALT (Jochemsen and Jeurissen 2002).

Orally administrated vaccine must, therefore, survive a passage through the crop and stomach into the intestine. Protein antigens combined with OMV vaccines may thus benefit from an additional protection, e.g., liposome encapsulation.

It is not certain whether the booster should follow the same route. Several studies have tested various administration routes of OMV vaccines in other animals. Schild et al. (2008) immunized mice with OMVs derived from Vibrio cholerae via three routes (intranasal, intragastric, and intraperitoneal). Interestingly, all the routes studied had comparably strong effect on Ig titers (IgG1, IgG2, IgM, and IgA were studied) and provided comparable protection against $V$. cholerae infection and colonization. The intranasal and intragastric immunized mice were fully protected, and from intraperitoneal immunized mice, at least 10,000-fold lower numbers of Vibrio CFU were recovered than in the infection of naive animals. Nieves et al. (2011) demonstrated that a subcutaneous immunization with OMVs isolated from Burkholderia pseudomallei provides a significant protection against lethal B. pseudomallei infection in BALB/c mice.

Thus, the oral route for booster in chicken may be preferred as the simplest one, but further studies are needed to verify and compare its efficacy to other modes of delivery.

In conclusion, we presented a proof-of-concept study on the usability of $C$. jejuni OMVs enriched with CjaA antigen as an in ovo protective vaccine against $C$. jejuni in chickens. While the response after single dose of a vaccine is clearly observable, the effect was rather short lived. Further studies on booster dosing and/or immunoadjuvant support are required.

Acknowledgments We thank Dr. L. Trzeciak for his critical reading of the manuscript and for assistance in statistical analysis.

Compliance with ethical standards All animal experiments were carried out with approval nr 397/2012 of the LKE (First Local Ethical Committee on Animal Testing in Warsaw). All applicable international, national, and institutional guidelines for the care and use of animals were followed.

Funding The work was supported by grants from PARENT BRIDGE program of the Foundation for Polish Science, cofinanced by the European Union under the European Regional Development Fund, POMOST/2012-6/4.

Conflict of interest The authors declare that they have no conflict interest.

Open Access This article is distributed under the terms of the Creative Commons Attribution 4.0 International License (http:// creativecommons.org/licenses/by/4.0/), which permits unrestricted use, distribution, and reproduction in any medium, provided you give appropriate credit to the original author(s) and the source, provide a link to the Creative Commons license, and indicate if changes were made. 


\section{References}

Acevedo R, Fernandez S, Zayas C, Acosta A, Sarmiento ME, Ferro VA, Rosenquist E, Campa C, Cardoso D, Garcia L, Perez JL (2014) Bacterial outer membrane vesicles and vaccine applications. Front Immunol 5:121. doi:10.3389/fimmu.2014.00121

Asensio CJ, Gaillard ME, Moreno G, Bottero D, Zurita E, Rumbo M, van der Ley P, van der Ark A, Hozbor D (2011) Outer membrane vesicles obtained from Bordetella pertussis Tohama expressing the lipid a deacylase PagL as a novel acellular vaccine candidate. Vaccine 29(8):1649-1656. doi:10.1016/j.vaccine.2010.12.068

Bande F, Arshad SS, Bejo MH, Moeini H, Omar AR (2015) Progress and challenges toward the development of vaccines against avian infectious bronchitis. J Immunol Res 2015:424860. doi:10.1155/2015/424860

Bar-Shira E, Sklan D, Friedman A (2003) Establishment of immune competence in the avian GALT during the immediate post-hatch period. Dev Comp Immunol 27(2):147-157

Brisbin JT, Gong J, Sharif S (2008) Interactions between commensal bacteria and the gut-associated immune system of the chicken. Anim Health Res Rev 9(1):101-110. doi:10.1017/S146625230800145X

Buckley AM, Wang J, Hudson DL, Grant AJ, Jones MA, Maskell DJ, Stevens MP (2010) Evaluation of live-attenuated Salmonella vaccines expressing Campylobacter antigens for control of $C$. jejuni in poultry. Vaccine 28(4):1094-1105. doi:10.1016/j. vaccine.2009.10.018

Carter NJ (2013) Multicomponent meningococcal serogroup B vaccine $(4 \mathrm{CMenB}$; Bexsero $((\mathrm{R})))$ : a review of its use in primary and booster vaccination. BioDrugs 27(3):263-274. doi:10.1007/s40259-0130029-2

Chutkan H, Macdonald I, Manning A, Kuehn MJ (2013) Quantitative and qualitative preparations of bacterial outer membrane vesicles. Methods Mol Biol 966:259-272. doi:10.1007/978-1-62703-245-2 16

Clark JD, Oakes RD, Redhead K, Crouch CF, Francis MJ, Tomley FM, Blake DP (2012) Eimeria species parasites as novel vaccine delivery vectors: anti-Campylobacter jejuni protective immunity induced by Eimeria tenella-delivered CjaA. Vaccine 30(16):2683-2688. doi:10.1016/j.vaccine.2012.02.002

Collins BS (2011) Gram-negative outer membrane vesicles in vaccine development. Discov Med 12(62):7-15

Conlan AJ, Coward C, Grant AJ, Maskell DJ, Gog JR (2007) Campylobacter jejuni colonization and transmission in broiler chickens: a modelling perspective. J R Soc Interface 4(16):819 829. doi:10.1098/rsif.2007.1015

Cordwell SJ, Len AC, Touma RG, Scott NE, Falconer L, Jones D, Connolly A, Crossett B, Djordjevic SP (2008) Identification of membrane-associated proteins from Campylobacter jejuni strains using complementary proteomics technologies. Proteomics $8(1)$ : 122-139. doi:10.1002/pmic.200700561

Davis L, Young K, DiRita V (2008) Genetic manipulation of Campylobacter jejuni. Curr Protoc Microbiol Chapter 8: Unit 8A 2 1-8A 217 doi:10.1002/9780471729259.mc 08a02s10

EFSA, ECDC (2015) The European Union summary report on trends and sources of zoonoses, zoonotic agents and foodborne outbreaks in 2013. EFSA J 2015 13(1):3991. doi:10.2903/j.efsa.2015.3991

Elmi A, Watson E, Sandu P, Gundogdu O, Mills DC, Inglis NF, Manson E, Imrie L, Bajaj-Elliott M, Wren BW, Smith DG, Dorrell N (2012) Campylobacter jejuni outer membrane vesicles play an important role in bacterial interactions with human intestinal epithelial cells. Infect Immun 80(12):4089-4098. doi:10.1128/IAI.00161-12

Giuliani MM, Adu-Bobie J, Comanducci M, Arico B, Savino S, Santini L, Brunelli B, Bambini S, Biolchi A, Capecchi B, Cartocci E, Ciucchi L, Di Marcello F, Ferlicca F, Galli B, Luzzi E, Masignani V, Serruto D, Veggi D, Contorni M, Morandi M, Bartalesi A, Cinotti V, Mannucci D, Titta F, Ovidi E, Welsch JA, Granoff D, Rappuoli R, Pizza M (2006) A universal vaccine for serogroup B meningococcus. Proc Natl Acad Sci U S A 103(29):10834-10839. doi:10.1073/pnas.0603940103

Havelaar AH, Mangen MJ, de Koeijer AA, Bogaardt MJ, Evers EG, Jacobs-Reitsma WF, van Pelt W, Wagenaar JA, de Wit GA, van der Zee H, Nauta MJ (2007) Effectiveness and efficiency of controlling Campylobacter on broiler chicken meat. Risk Anal 27(4):831844. doi:10.1111/j.1539-6924.2007.00926.x

Holmes K, Mulholland F, Pearson BM, Pin C, McNicholl-Kennedy J, Ketley JM, Wells JM (2005) Campylobacter jejuni gene expression in response to iron limitation and the role of fur. Microbiology 151(Pt 1):243-257. doi:10.1099/mic.0.27412-0

Holst J, Martin D, Arnold R, Huergo CC, Oster P, O’Hallahan J, Rosenqvist E (2009) Properties and clinical performance of vaccines containing outer membrane vesicles from Neisseria meningitidis. Vaccine 27(Suppl 2):B3-12. doi:10.1016/j.vaccine. $\% 232009.04 .071$

Jang KS, Sweredoski MJ, Graham RL, Hess S, Clemons WM Jr (2014) Comprehensive proteomic profiling of outer membrane vesicles from Campylobacter jejuni. J Proteome 98:90-98. doi:10.1016/j. jprot.2013.12.014

Jochemsen P, Jeurissen SH (2002) The localization and uptake of in ovo injected soluble and particulate substances in the chicken. Poult Sci 81(12): 1811-1817

Kaakoush NO, Castano-Rodriguez N, Mitchell HM, Man SM (2015) Global epidemiology of Campylobacter infection. Clin Microbiol Rev 28(3):687-720. doi:10.1128/CMR. 00006-15

Kirkpatrick BD, Tribble DR (2011) Update on human Campylobacter jejuni infections. Curr Opin Gastroenterol 27(1):1-7. doi:10.1097 /MOG.0b013e3283413763

Korlath JA, Osterholm MT, Judy LA, Forfang JC, Robinson RA (1985) A point-source outbreak of campylobacteriosis associated with consumption of raw milk. J Infect Dis 152(3):592-596

Kuehn MJ, Kesty NC (2005) Bacterial outer membrane vesicles and the host-pathogen interaction. Genes Dev 19(22):2645-2655. doi:10.1101/gad.1299905

Layton SL, Morgan MJ, Cole K, Kwon YM, Donoghue DJ, Hargis BM, Pumford NR (2011) Evaluation of Salmonella-vectored Campylobacter peptide epitopes for reduction of Campylobacter jejuni in broiler chickens. Clin Vaccine Immunol 18(3):449-454. doi:10.1128/CVI.00379-10

Lindmark B, Rompikuntal PK, Vaitkevicius K, Song T, Mizunoe Y, Uhlin BE, Guerry P, Wai SN (2009) Outer membrane vesicle-mediated release of cytolethal distending toxin (CDT) from Campylobacter jejuni. BMC Microbiol 9:220. doi:10.1186/1471-2180-9-220

Mangen MJ, Havelaar AH, Poppe KP, de Wit GA (2007) Cost-utility analysis to control Campylobacter on chicken meat: dealing with data limitations. Risk Anal 27(4):815-830. doi:10.1111/j.15396924.2007.00925.x

Martin NG, Snape MD (2013) A multicomponent serogroup B meningococcal vaccine is licensed for use in Europe: what do we know, and what are we yet to learn? Expert Rev Vaccines 12(8):837-858. doi:10.1586/14760584.2013.814862

Mashburn LM, Whiteley M (2005) Membrane vesicles traffic signals and facilitate group activities in a prokaryote. Nature 437(7057):422425. doi: 10.1038 /nature 03925

Mashburn-Warren L, Howe J, Garidel P, Richter W, Steiniger F, Roessle M, Brandenburg K, Whiteley M (2008) Interaction of quorum signals with outer membrane lipids: insights into prokaryotic membrane vesicle formation. Mol Microbiol 69(2):491-502

Mast J, Goddeeris BM (1999) Development of immunocompetence of broiler chickens. Vet Immunol Immunopathol 70(3-4):245-256

Muller A, Thomas GH, Horler R, Brannigan JA, Blagova E, Levdikov VM, Fogg MJ, Wilson KS, Wilkinson AJ (2005) An ATP-binding cassettetype cysteine transporter in Campylobacter jejuni inferred from the structure of an extracytoplasmic solute receptor protein. Mol Microbiol 57(1):143-155. doi:10.1111/j.1365-2958.2005.04691.x 
Negash T, SO a-G, Gruys E (2004) Comparison of in ovo and post-hatch vaccination with particular reference to infectious bursal disease. A review. Vet Q 26(2):76-87. doi:10.1080/01652176.2004.9695170

Newell DG, Fearnley C (2003) Sources of Campylobacter colonization in broiler chickens. Appl Environ Microbiol 69(8):4343-4351

Nieves W, Asakrah S, Qazi O, Brown KA, Kurtz J, Aucoin DP, McLachlan JB, Roy CJ, Morici LA (2011) A naturally derived outer-membrane vesicle vaccine protects against lethal pulmonary Burkholderia pseudomallei infection. Vaccine 29(46):8381-8389. doi:10.1016/j.vaccine.2011.08.058

Pawelec D, Rozynek E, Popowski J, Jagusztyn-Krynicka EK (1997) Cloning and characterization of a Campylobacter jejuni 72Dz/92 gene encoding a $30 \mathrm{kDa}$ immunopositive protein, component of the ABC transport system; expression of the gene in avirulent Salmonella typhimurium. FEMS Immunol Med Microbiol 19(2): $137-150$

Roberts R, Moreno G, Bottero D, Gaillard ME, Fingermann M, Graieb A, Rumbo M, Hozbor D (2008) Outer membrane vesicles as acellular vaccine against pertussis. Vaccine 26(36):4639-4646. doi:10.1016 /j.vaccine.2008.07.004

Sahin O, Luo N, Huang S, Zhang Q (2003) Effect of Campylobacterspecific maternal antibodies on Campylobacter jejuni colonization in young chickens. Appl Environ Microbiol 69(9):5372-5379

Schild S, Nelson EJ, Camilli A (2008) Immunization with Vibrio cholerae outer membrane vesicles induces protective immunity in mice. Infect Immun 76(10):4554-4563. doi:10.1128/IAI.00532-08

Sharma JM (1999) Introduction to poultry vaccines and immunity. Adv Vet Med 41:481-494

Shoaf-Sweeney KD, Larson CL, Tang X, Konkel ME (2008) Identification of Campylobacter jejuni proteins recognized by maternal antibodies of chickens. Appl Environ Microbiol 74(22): 6867-6875. doi:10.1128/AEM.01097-08

Simon R, Priefer U, Pühler A (1983) A broad host range mobilization system for in vivo genetic engineering: transposon mutagenesis in gram negative bacteria. Nat Biotechnol 1:784-791. doi:10.1038 /nbt1183-784

Toro H, Tang DC, Suarez DL, Sylte MJ, Pfeiffer J, Van Kampen KR (2007) Protective avian influenza in ovo vaccination with nonreplicating human adenovirus vector. Vaccine 25(15):2886-2891. doi:10.1016/j.vaccine.2006.09.047

van der Pol L, Stork M, van der Ley P (2015) Outer membrane vesicles as platform vaccine technology. Biotechnol J 10(11):1689-1706. doi:10.1002/biot.201400395

Vegge CS, Brondsted L, Ligowska-Marzeta M, Ingmer H (2012) Natural transformation of Campylobacter jejuni occurs beyond limits of growth. PLoS One 7(9):e45467. doi:10.1371/journal.pone.0045467

Wyszynska A, Raczko A, Lis M, Jagusztyn-Krynicka EK (2004) Oral immunization of chickens with avirulent Salmonella vaccine strain carrying $C$. jejuni 72Dz/92 cjaA gene elicits specific humoral immune response associated with protection against challenge with wild-type Campylobacter. Vaccine 22(11-12):1379-1389. doi:10.1016/j.vaccine.2003.11.001

Wyszynska A, Zycka J, Godlewska R, Jagusztyn-Krynicka EK (2008) The Campylobacter jejuni/coli cjaA (cj0982c) gene encodes an Nglycosylated lipoprotein localized in the inner membrane. Curr Microbiol 57(3):181-188. doi:10.1007/s00284-008-9171-3

Yao R, Alm RA, Trust TJ, Guerry P (1993) Construction of new Campylobacter cloning vectors and a new mutational cat cassette. Gene 130(1):127-130 